\title{
Evaluación de parámetros de crecimiento en plántulas de Cedrela odorata bajo diferentes condiciones hídricas y su adaptación en sustratos degradados
}

\author{
Jordi Altimira $^{1}$ \\ Anaïs Arnal ${ }^{1}$ \\ Marc Boada ${ }^{1}$ \\ Cristian Casanova ${ }^{1}$ \\ Jordi Herrero $^{1}$ \\ Martí Boada ${ }^{1}$ \\ Alejandrina Herrera ${ }^{2}$ \\ Verónica Ruiz ${ }^{2}$
}

\section{RESUMEN}

La desforestación del bosque seco tropical se ha convertido en uno de los mayores problemas ambientales de Nicaragua de las últimas décadas. Esta pérdida de masas boscosas ha supuesto una reducción de los recursos naturales esenciales para la población, tales como madera, alimentos y agua. La elevada explotación de Cedrela odorata (cedro real) para su aprovechamiento maderable ha situado a esta especie como vulnerable en la lista roja de la IUCN. Este estudio pretende brindar información sobre la capacidad de $C$. odorata de crecer en sustratos degradados y bajo condiciones hídricas eventualmente diferentes, resultantes del cambio climático. Para ello se evaluó el efecto de dos factores sobre la germinación y el crecimiento de plántulas de $C$. odorata, siendo estos: tres tipos de sustrato y cinco cantidades de agua. Los sustratos que se seleccionaron fueron de finca ganadera, de finca agrícola y de bosque seco tropical (control). Los riegos establecidos fueron, en base a la precipitación media del rodal: 50\%, 75\%, 100\% (control), 150\% y 200\%. En total, se establecieron quince tratamientos combinación de los dos factores. Los resultados muestran que $C$. Odorata tiene buena capacidad para crecer en sustratos degradados con pluviometrías de alrededor de $1200 \mathrm{~mm} /$ año y hasta un 50\% inferiores. C. Odorata tiene dificultades para germinar con pluviometrías superiores a las actuales, especialmente en suelos con escassa capacidad de infiltración. El crecimiento de C. odorata con pluviometrías superiores a los $1200 \mathrm{~mm} /$ año presenta un insuficiente desarrollo radicular que le dificultaría la supervivencia durante la estación seca.

Palabras clave: reforestación, cambio climático, estrés hídrico, desarrollo radicular, área foliar, anegamiento.

Recibido: 2 de diciembre de 2014

Aceptado: 25 de junio de 2015

1 Universidad Autónoma de Barcelona (UAB)

2 Facultad Regional Multidisciplinaria de Estelí - Universidad Nacional Autónoma de Nicaragua. Docentes investigadoras. Correo de contacto: verolisrg@gmail.com 


\title{
Evaluation of growth parameters in Cedrela odorata seedlings under different water conditions and their adaptation to degraded substrates
}

\begin{abstract}
Deforestation of tropical dry forest has become a major environmental problems in Nicaragua in the last few decades. This loss of forested areas has reduced the natural resources essential to the population, such as wood, food and water. The high exploitation of Cedrela odorata (real cedar) for timber harvesting has placed this species as vulnerable in the IUCN Red List. This study aims to provide information about the ability of $C$. odorata of growing in degraded substrates and under water conditions eventually different resulting from climate change. For this, the effect of two factors on germination and seedling growth of $C$. odorata was evaluated, being these: three types of substrate and five volumes of water. The substrates were selected from livestock, agricultural farm and tropical dry forest (control). The risks were established, based on the rainfall of the stand: $50 \%, 75 \%, 100 \%$ (control), 150\% and 200\%. Overall, fifteen treatments combining the two factors were established. The results show that $C$. odorata has good ability to grow on degraded substrates with precipitation rates of about $1200 \mathrm{~mm}$ / year and up to $50 \%$ lower. C. odorata has difficulty germinating, especially in soils with scarce infiltration capacity precipitation rates. The growth of C. odorata in excess of $1200 \mathrm{~mm}$ / year precipitation rates presents insufficient root growth that would hinder survival during the dry season.
\end{abstract}

Keywords: reforestation, climate change, water stress, root growth, leaf area, waterlogging. 


\section{INTRODUCCIÓN}

El bosque seco tropical (BST) de la región centroamericana ha sufrido una elevada tasa de deforestación durante las últimas décadas, provocando problemas ambientales y sociales tanto a nivel local como regional. Sumado a la pérdida de suelos y a la fragmentación de hábitats, esto supone una importante amenaza para la biodiversidad vegetal y animal (Miles et al, 2006) calificó el bosque seco tropical como una de las regiones de bosque seco más amenazadas del planeta(Janzen, 1983).Este se ha visto transformado en gran medida en un agroecosistema formado por un mosaico de campos, pastizales y pequeños bosques secundarios donde sus posibilidades de regeneración están muy limitadas (Kramer, 1997).

El Cedrela odorata (cedro real) es una especie que forma parte del bosque seco tropical. Se trata de una especie nativa de Nicaragua y se encuentra en situación crítica a causa de la sobreexplotación a la que ha sido sometida debido al alto valor comercial de su madera(Miles et al., 2006). Actualmente se trata de una especie prioritaria a nivel nacional para ser utilizada en la reforestación de las zonas degradadas, debido a su catalogación en la lista roja de la UICN como especie vulnerable. Además, al ser una especie con potencial maderable, podría ser utilizada para fomentar la reforestación voluntaria en las fincas privadas para una posible explotación en un futuro de forma sostenible. Paralelamente a la problemática de la desforestación, las proyecciones para el cambio climático en Centroamérica estiman un probable incremento en la frecuencia de los episodios de lluvias intensas, acompañado de un aumento en las sequías (IPCC 2007). Esto supondría un incremento severo de los impactos y riesgos ambientales.

El presente estudio tiene como propósito evaluar el desarrollo de la primera fase de crecimiento del Cedrela odorata en los diferentes escenarios previstos por los modelos de cambio climático y determinar su viabilidad como especie útil para realizar la reforestación en sustratos degradados. Para ello se trabajó en objetivos específicos: evaluar la velocidad y porcentaje de germinación de las semillas de $C$. odorata y el desarrollo de las plántulas bajo diferentes condiciones hídricas y en distintos sustratos posibles.

\section{MATERIALES Y MÉTODOS}

\section{Ubicación del área de estudio}

El estudio se realizó en las instalaciones de la Estación Experimental para el Estudio del Trópico Seco El Limón de la UNAN-MANAGUA FAREMEstelí, en la Región Norte-central de Nicaragua (UTM 16P 0568786, 1444044) a $865 \mathrm{msnm}$.

\section{Diseño del estudio}

Se evaluó el crecimiento y desarrollo de $C$. odorata en sustratos degradados y bajo diferentes condiciones hídricas, se establecieron las dos variables independientes a tratar: tipo de sustrato y pluviometría. Se eligieron tres tipos de sustratos diferentes, uno extraído de un bosque seco tropical que se utilizó como control, uno de una finca agrícola y otro de una finca de ganadería, ambos de zonas degradadas en las que antiguamente se alzaba el bosque seco tropical y en las cuales puede ser necesaria una reforestación en un futuro.

Para establecer las dosis de agua a las que se iban a someter las plantas se utilizaron los datos de pluviometría de los meses lluviosos desde abril a noviembre del periodo 2009 - 2012 de la estación meteorológica de la Estación "El Limón”. Mediante la frecuencia de precipitaciones de estos meses se estableció la frecuencia y el calendario de riego. Seguidamente, se calculó la cantidad de riego equivalente a la media de precipitación diaria en los meses lluviosos y se establecieron cinco grupos simulando diferentes pluviometrías: 50\%, 75\%, 100\%, 
$150 \%$ y $200 \%$ de la pluviometría actual registrada en la estación. Las variaciones en la pluviometría se basan en la incertidumbre de las previsiones de los futuros modelos de precipitación del cambio climático.

Con tres sustratos distintos y cinco riegos diferentes se obtuvo un total de quince tratamientos.

\section{Montaje del experimento}

Se realizó un tratamiento pregerminativo a las semillas de Cedrela odorata, obtenidas del stock de la Estación "El Limón" almacenadas durante 2 años a una temperatura de $3^{\circ} \mathrm{C}$. El porcentaje de germinación que se obtuvo de este tratamiento fue del $83 \%$,

Para determinar la tasa y la velocidad de germinación de las semillas de $C$. orodata bajo condiciones de estrés hídrico en distintos tipos de sustrato se llenaron 30 bolsas de vivero de polietileno ( $\varnothing=8 \mathrm{~cm}$ prof. $18 \mathrm{~cm}$ ) para cada tratamiento, con el resultado de un total de 450 bolsas. Posteriormente se colocaron las bolsas debidamente rotuladas y separadas por tratamientos en mesas de 1,2 $\mathrm{m}$ de altura en el centro del invernadero. Se colocaron mantas absorbentes debajo de las bolsas.

Con el resultado obtenido en el tratamiento pregerminativo, se determinó que para asegurar un número suficiente de plántulas germinadas bastaría con duplicar el número de semillas, de manera que se plantaron 2 semillas por bolsa a una profundidad de $0,5 \mathrm{~cm}$., obteniendo un total de 900 semillas para garantizar la germinación de 450 .

El estudio se realizó durante los meses de octubre a enero de 2013-2014, con rangos de temperaturas de $20^{\circ} \mathrm{C}$ a $30^{\circ} \mathrm{C}$ y humedad relativa de $40 \%$ a $95 \%$.

Para la dosificación del riego se construyeron distintos recipientes a partir de recipientes de plástico reutilizados. Con estos se regó manualmente bolsa por bolsa los días establecidos en el calendario de riego con agua del pozo de la Estación "El Limón".
Los primeros días del ensayo, se hizo un seguimiento diario de las semillas para identificar aquellas que germinaban, permitiendo así calcular la tasa de germinación y el T50, que es el número de días que se tarda en alcanzar el $50 \%$ de la germinación total (Narbona, Arista, \& Ortiz, 2003) (Bewley \& Black, n.d.)

\section{Evaluación del crecimiento y biomasa}

Transcurridas dos semanas se trasplantaron plántulas de las bolsas en las que había germinado más de una semilla a aquellas en las que no había crecido ninguna, por tal de disponer de suficientes muestras para la medición de las variables y parámetros descritos más adelante.

Después de regar las plántulas durante 2 meses se procedió a evaluar la supervivencia, la producción y distribución de biomasa en los 15 tratamientos.

Se seleccionaron al azar 10 plántulas de cada tratamiento y se midió la parte aérea y subterránea de cada una para cuantificar las diferencias en el crecimiento de $C$. odorata de los distintos tratamientos. A cada plántula muestreada se le separó el sistema radicular de la parte aérea y las hojas del tallo. Se midió con una regla milimetrada la longitud del tallo y la longitud de la raíz principal. También se contó el número de hojas y se midió el área foliar. Esta última se calculó en cinco muestras elegidas de forma aleatoria de cada tratamiento, a las que se les escanearon todas sus hoja sutilizando un escáner marca hp modelo scanjet 5590 y se calculó la superficie foliar con el programa imagen (Guerrero, Andrés, Quintero, \& Pérez, 2012).

Para el estudio de la distribución de la biomasa de las plántulas se separaron hojas, tallo y raíz y se secaron con un horno marca EIT modelo CIEC-EQL-04 durante 48 horas a una temperatura de $80^{\circ} \mathrm{C}$. Posteriormente, se pesaron por separado los pesos secos de la raíz, tallo y hojas, utilizando una balanza analítica marca Acculab modelo VIC-303. 
Obtenidos todos los datos, se procedió a calcular diferentes índices para poder obtener información sobre el crecimiento y desarrollo de las plántulas y hacer comparaciones entre los diferentes tratamientos. Los índices calculados fueron: relación entre biomasa seca aérea y biomasa seca radical (RBAR), área foliar especifica (AFE) y relación peso foliar (RPF).

\section{Análisis estadístico}

Los datos de porcentaje de germinación (PGT) y la velocidad de germinación (T50 en días) no presentaron una distribución normal. Debido a esto se efectuó un análisis de la varianza no paramétrico Kruskal-Wallis al 5\%, con el programa INFOSTAT (2008).

En el caso de los datos de Longitud de Raíz (LR), Peso Seco Total (PST), Relación Biomasa Aérea entre Biomasa Radicular (RBAR),Altura del tallo (AT) y relación peso foliar (RPF) fueron sometidos al Análisis de Varianza (ANOVA) utilizando el método Análisis de Varianza para un Bifactorial en DCA (Pedroza \& Dicovskyi, 2007) y posteriormente se les aplicó la prueba SNK por medio del programa SPSSv.20. Para las variables LR, PST, y RBAR fue necesario transformar los datos para que estos presentaran una distribución normal antes de ser analizados, dichas transformaciones fueron, y respectivamente.

\section{RESULTADOS}

\section{Porcentaje de germinación}

Se hallaron diferencias significativas entre los tratamientos con diferentes riegos $(p<0,05)$.El porcentaje de germinación menor se encontró en el los tratamientos con 98ml (Tabla 1) (A)

En cuanto al efecto del "sustrato" en el porcentaje de germinación, solo se hallaron diferencias significativas entre los sustratos agrícola y ganadero. Se observó que el porcentaje de germinación fue inferior en los tratamientos con sustrato agrícola respecto a los que usaban sustrato ganadero (Tabla 1) (B)

Los porcentajes finales de germinación más bajos se obtuvieron en los tratamientos que usaban sustrato agrícola y riego de $98 \mathrm{ml}$, en los cuales el porcentaje final de germinación estuvo alrededor del 10\%. En el caso del sustrato ganadero se observaron porcentajes de germinación más bajos en los tratamientos con riego $98 \mathrm{ml}$ que en los tratamientos con riegos inferiores.

\section{Velocidad de germinación (T50)}

La T50 en función del riego aplicado en los tratamientos, presentó diferencias significativas $(p<0,05)$. La mayor velocidad de germinación se obtuvo en los tratamientos con riego de $98 \mathrm{ml}$. Por el contrario, la menor velocidad de germinación se obtuvo con el riego de $24 \mathrm{ml}$ (Tabla 2) (A)Tabla 2. Aunque significativas, las diferencias observadas fueron mínimas. El efecto del sustrato en la T50 no presento diferencias significativas (Tabla 2) (B).

\section{Supervivencia}

Todos los tratamientos presentaron una supervivencia de plántulas del $100 \%$, exceptuando el tratamiento $98 \mathrm{~A}$, en el cual hubo una mortalidad del $17 \%$.

\section{Parámetros de crecimiento}

Se observaron diferencias significativas entre los sustratos bosque y agrícola, la longitud del tallo fue superior en los tratamientos con sustrato de bosque respecto a los tratamientos con sustrato agrícola (Tabla 4).

Aunque no se observaron diferencias significativas en este parámetro en función del riego (Tabla 3), si se pudieron observar en la interacción de las variables independientes sustrato y riego $(\mathrm{P}<0,05)$. El tratamiento 98A fue el que presento longitudes 
significativamente más bajas, no diferenciándose de forma significativa únicamente con el tratamiento 37. Los demás tratamientos no presentaron diferencias significativas entre ellos. (Ilustración1)

La longitud de la raíz presentó diferencias significativas en los tratamientos que usaban respeto a los que usaban riegos inferiores. Se observó una tendencia a menores longitudes a medida que aumentaba el riego (Tabla 3). Este mismo parámetro en función del sustrato, presentó valores significativamente más altos en el sustrato de bosque (Tabla 4), no se observaron diferencias significativas en la interacción de las dos variables independientes.

El peso seco total (PST) presentó diferencias significativas en función de la cantidad de riego entre los tratamientos con riego 24 y control (49 ml) respecto a los tratamientos con riegos de 37, 74 y 98 ml. Los valores más altos de PST fueron observados en el tratamiento control y el de riego $24 \mathrm{ml}$ (Tabla 3). El sustrato ganadero presento valores significativamente más altos de PST que los demás sustratos (Tabla 4).

Se observaron diferencias significativas en la interacción de las variables riego y sustrato $(\mathrm{P}<0,05)$. El tratamiento 98A presento valores significativamente más bajos que los demás exceptuando el tratamiento 74B. Por otra parte, los tratamientos 74B, 74A, 37A y $98 \mathrm{~B}$ presentaron valores significativamente más bajos que los tratamientos 98G, 24G, 49G y 49A (Ilustración2).

La relación entre la biomasa aérea y la radicular (RBAR) en función de las dosis de riego presentó diferencias significativas entre todos los tratamientos, exceptuando los riegos de 24 y $37 \mathrm{ml}$ que no presentaron diferencias entre sí. Pudo observarse una tendencia a valores mayores de RBAR a medida que aumentaba la cantidad de riego (Tabla 3 ).
Esta misma relación en función del sustrato presentó diferencias significativas en el sustrato de bosque respecto a los otros dos, siendo éste el que presentó un valor más bajo (Tabla 4).

Se observaron diferencias significativas en la interacción de las variables independientes sustrato y riego $(\mathrm{P}<0,05)$. Los tratamientos $24 \mathrm{~B}$ y $37 \mathrm{G}$ presentaron los valores significativamente más de RBAR. Los tratamientos 98A y 98G presentaron valores significativamente más altos que todos los demás excepto el tratamiento $74 \mathrm{~A}$, con el cual no tuvieron diferencias significativas. La tendencia general de la RBAR estuvo marcada principalmente por la cantidad de riego, se observó una correlación positiva entre el riego y la RBAR (Ilustración3).

En la Relación peso foliar (RPF) en función del riego se observaron diferencias significativas entre todos los tratamientos, exceptuando el de riego $37 \mathrm{ml}$, que no presento diferencias con los tratamientos de 24 y $49 \mathrm{ml}$. El valor de este parámetro creció a medida que aumentaba la cantidad de riego (Tabla 3 ).

Este mismo parámetro en función del sustrato solo presentó diferencias significativas en el sustrato de bosque, siendo éste el que presento valores más bajos (Tabla 4).

En la Área foliar específica (AFE) en función del riego se observaron diferencias significativas entre el tratamiento control $(49 \mathrm{ml})$ y todos los demás tratamientos con riegos diferentes. Los tratamientos realizados con riego $24 \mathrm{ml}$ presentaron diferencias significativas con los tratamientos con riego 37,49 y $74 \mathrm{ml}$.

Los valores más bajos de AFE se obtuvieron en el tratamiento control. Mientras que los tratamientos con riego de $24 \mathrm{ml}$ presentaron valores significativamente inferiores a los tratamientos con riego $37 \mathrm{ml} \mathrm{y} 74 \mathrm{ml}$ y significativamente superiores al tratamiento control. 
Este mismo parámetro en función del sustrato presentó diferencias significativas entre cada uno de los sustratos, presentando valores superiores en el sustrato ganadero e inferiores en el sustrato de bosque.

riego $24 \mathrm{ml}$ respecto al resto de tratamientos con diferentes riegos. También se observaron diferencias significativas en los tratamientos con riegos de $74 \mathrm{ml}$ y $98 \mathrm{ml}$

\section{DISCUSIÓN}

El porcentaje de germinación presentó valores especialmente bajos en el tratamiento 98A (10\%), en el cual se observó anegamiento del sustrato durante los riegos realizados, lo que dificultó la germinación de las semillas(Bewley \& Black, 1978).

Los valores de T50, aunque fueron menores en los riegos mayores, las diferencias fueron tan bajas que no se consideraron relevantes.

Se observó una mortalidad del 17\% en el tratamiento 98A debido al anegamiento del suelo. Ya que el exceso de agua en el suelo provoca desordenes fisiológicos que alteran el desarrollo normal de la planta (Pardos, 2004).

La longitud del tallo solo presentó diferencias significativas en función del sustrato, obteniendo valores mayores en el sustrato del bosque. Esto podría deberse a una mayor disponibilidad de nutrientes en el sustrato de bosque como consecuencia de un $\mathrm{pH}$ menor (Ruano, M \& J.R, 2003)además de un mejor drenaje de agua (Holdridge \& R, 1979) ambas condiciones han demostrado que favorecen un mejor desarrollo del $C$. odorata.

Se observó una tendencia a longitudes de raíz más pequeñas en riegos más altos. Este resultado concuerda con el caso del RBAR y RPF, donde pudo observarse una tendencia a valores más altos en riegos más altos.
Esto significa que las diferencias en los valores de estos parámetros están determinadas principalmente por la variación de la biomasa radicular, que fue la más afectada por las diferentes cantidades de riego. Relacionando estos parámetros con el PST pudo observarse que a medida que disminuyó la biomasa radicular de las plántulas estas no destinaron más biomasa al desarrollo de la parte aérea.

Los resultados de estos parámetros indican que la planta no se desarrolla mejor con riegos tan altos, contrariamente a lo esperado. Además, teniendo en cuenta la estacionalidad de Nicaragua, un desarrollo pobre en las raíces como en los observados en tratamientos con riegos de 74 y $98 \mathrm{ml}$ causaría mortalidad en la época seca. En estos parámetros el efecto del sustrato no tuvo tanta importancia como el efecto del riego, aunque en los riegos problemáticos (74 y $98 \mathrm{ml}$ ), el sustrato bosque presentó distribuciones de biomasa más equilibradas que los otros sustratos, lo cual beneficiaria a las plántulas de cara a la supervivencia en la época seca.

Los valores observados en la AFE no se correspondieron a lo planteado en las hipótesis del estudio, sin embargo, sugieren que no hubo estrés hídrico en los riegos menores. Esta conclusión se vio confirmada en la longitud de raíz, puesto que no se encontraron valores bajos en riegos de 24 y $37 \mathrm{ml}$.

\section{CONCLUSIONES}

Cedrela odorata es una especie con potencial para reforestar zonas degradadas, pues se ha observado que es capaz de crecer en variedad de sustratos en las condiciones de pluviometría actuales y hasta un 50\% inferiores, sin presentar síntomas de estrés hídrico ni desequilibrios en el crecimiento.

En el caso de que el cambio climático causara un aumento en la intensidad de los episodios de lluvia, la especie Cedrela odorata podría tener problemas 
para sobrevivir y desarrollarse, pues los resultados encontrados en la longitud de la raíz, RPF y RBAR mostraron un desarrollo bajo del sistema radicular en las plántulas que crecieron en los tratamientos con riegos más altos. A raíz de esto, con la llegada de la estación seca, las plantas no tendrían suficiente capacidad para obtener recursos hídricos.

Especialmente en sustratos agrícolas, se observó que pluviometrías por encima de las actuales $(1200 \mathrm{~mm} /$ año), pueden causar el anegamiento de este tipo de suelos, reduciendo el porcentaje de germinación y disminuyendo la calidad y las probabilidades de supervivencia de las plántulas.
Algunos de los parámetros estudiados (PST y AFE), mostraron un mejor desarrollo de las plántulas en el sustrato ganadero, seguido del sustrato de bosque. Sin embargo, el sustrato de ganadería cambió durante la recolección del mismo, puesto que éste se aireó y vio reducida su fuerte compactación original. Así pues, podría utilizarse Cedrela odorata para la reforestación en suelos ganaderos a los que se les practique un proceso similar. El estudio no permitió observar si esta especie funcionaria para realizar reforestación en suelos ganaderos que no hayan sido tratados.

\section{TABLAS Y FIGURAS}

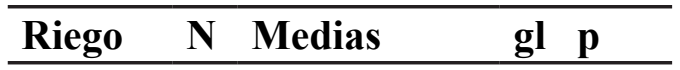

(A)

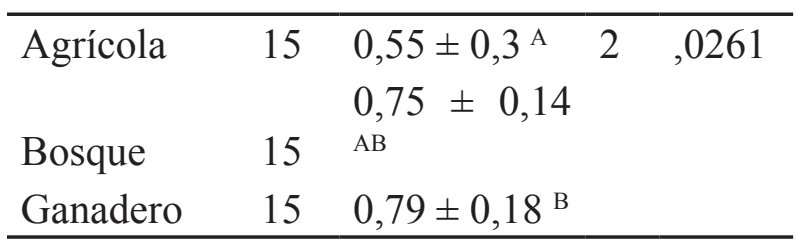

\begin{tabular}{lll}
\hline $24 \mathrm{ml}$ & 9 & $0,81 \pm 0,10^{\mathrm{BC}} 4,0010$ \\
$37 \mathrm{ml}$ & 9 & $0,81 \pm 0,09^{\mathrm{BC}}$ \\
$49 \mathrm{ml}$ & 9 & $0,81 \pm 0,21^{\mathrm{C}}$ \\
$74 \mathrm{ml}$ & 9 & $0,62 \pm 0,21^{\mathrm{AB}}$ \\
$98 \mathrm{ml}$ & 9 & $0,43 \pm 0,28^{\mathrm{A}}$ \\
\hline
\end{tabular}

Tabla 1: Germinación en función del riego (A) y en función del sustrato (B)

*Los tratamientos sin letras en común representan diferencias significativas.

\begin{tabular}{|c|c|c|c|c|c|c|c|c|c|c|c|}
\hline Riego & $\mathbf{N}$ & Medias (días) & gl & $\mathbf{p}$ & (A) & Sustrato & $\mathbf{N}$ & Medias (días) & gl & $\mathbf{P}$ & (B) \\
\hline $24 \mathrm{ml}$ & 9 & $9,06 \pm 0,37^{\mathrm{C}}$ & 4 & ,0023 & & Agrícola & 15 & $8,57 \pm 0,33$ & 2 & ,201 & \\
\hline $37 \mathrm{ml}$ & 9 & $8,73 \pm 0,38 \mathrm{ABC}$ & & & & Bosque & 15 & $8,86 \pm 0,44$ & & & \\
\hline $49 \mathrm{ml}$ & 9 & $8,73 \pm 0,24 \mathrm{BC}$ & & & & Ganadero & 15 & $8,69 \pm 0,44$ & & & \\
\hline $74 \mathrm{ml}$ & 9 & $8,52 \pm 0,40 \mathrm{AB}$ & & & & & & & & & \\
\hline $98 \mathrm{ml}$ & 9 & $8,47 \pm 0,46^{\mathrm{A}}$ & & & & & & & & & \\
\hline
\end{tabular}

Tabla 2: T50 en función del riego(A) y en función del sustrato (B)

*Los tratamientos sin letras en común representan diferencias significativas.

\begin{tabular}{|c|c|c|c|c|c|c|}
\hline Variables & $24 \mathrm{ml}$ & $37 \mathrm{ml}$ & $49 \mathrm{ml}$ (Control) & $74 \mathrm{ml}$ & $98 \mathrm{ml}$ & p \\
\hline LT (cm) & $\begin{array}{l}7,407 \pm \\
0,190^{\mathrm{NS}}\end{array}$ & $\begin{array}{c}7,197 \pm \\
0,190 \\
\text { NS }\end{array}$ & $\begin{array}{c}7,473 \pm 0,190 \\
\text { NS }\end{array}$ & $\begin{array}{c}7,737 \pm \\
0,190 \\
\text { NS }\end{array}$ & $\begin{array}{c}7,343 \pm \\
0,190 \\
\text { NS }\end{array}$ & ,363 \\
\hline Raíz LR(cm) & $\begin{array}{c}4,052 \pm \\
0.118 \\
\text { A }\end{array}$ & $\begin{array}{c}3,351 \pm \\
0.116 \\
\text { B }\end{array}$ & $\begin{array}{c}3,473 \pm 0.116 \\
\text { в }\end{array}$ & $\begin{array}{c}3,015 \pm \\
0.118\end{array}$ & $\begin{array}{c}2,818 \pm \\
0.118\end{array}$ & $<, 001$ \\
\hline
\end{tabular}




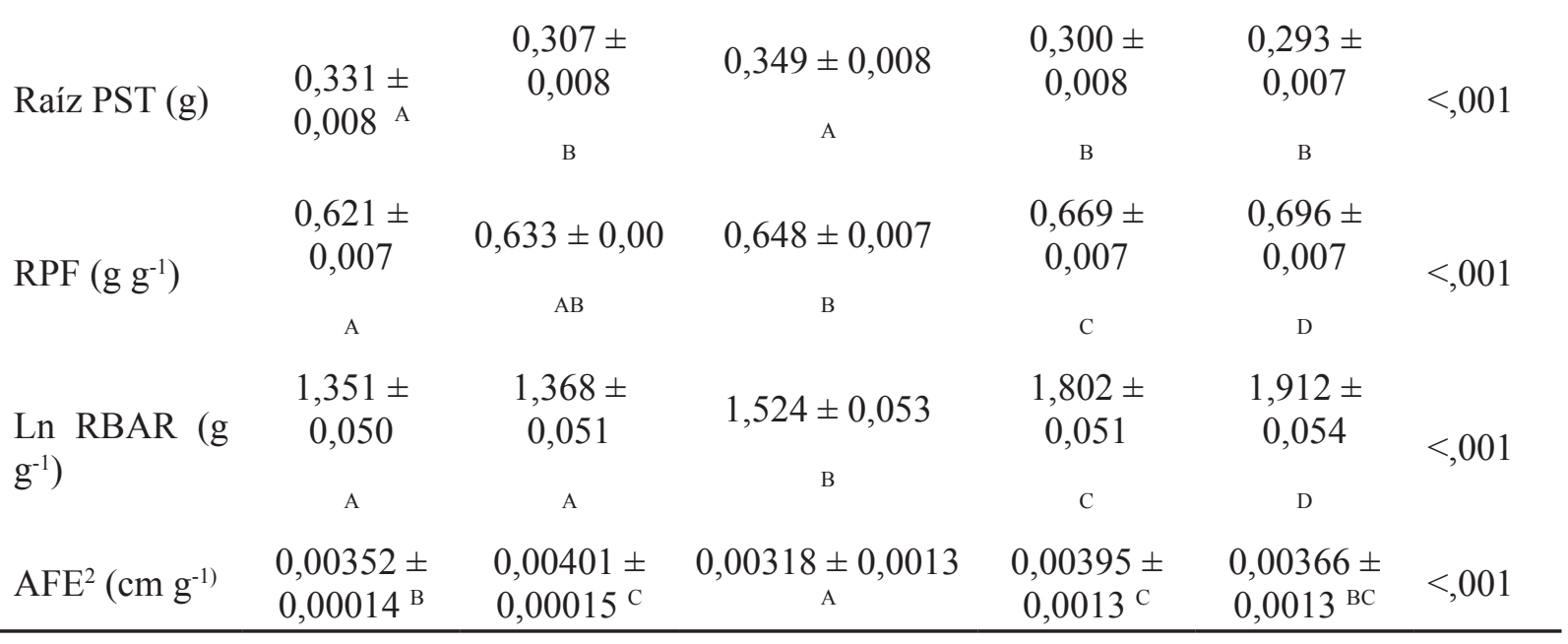

Tabla 3 Parámetros de crecimiento en función del riego

Diferentes letras indican diferencias significativas. LT Longitud tallo. LR Longitud raíz. PST Peso seco total, RPF Relación peso foliar, RBAR Relación biomasa aérea/ biomasa radicular, AFE Área foliar específica, (n= 30 , alfa $=0.05 \ldots)$

\begin{tabular}{lcccc}
\hline Variables & Ganadero & Bosque (Control) & Agrícola & P \\
\hline LT $(\mathrm{cm})$ & $7,478 \pm 0,147^{\mathrm{AB}}$ & $7,674 \pm 0,147^{\mathrm{B}}$ & $7,142 \pm 0,147^{\mathrm{A}}$ &, 039 \\
Raíz LR $(\mathrm{cm})$ & $3,360 \pm 0.092^{\mathrm{NS}}$ & $3,433 \pm 0,090^{\mathrm{NS}}$ & $3,235 \pm 0,090^{\mathrm{NS}}$ &, 291 \\
Raíz PST $(\mathrm{g})$ & $0,333 \pm 0,006^{\mathrm{B}}$ & $0,308 \pm 0,006^{\mathrm{A}}$ & $0,309 \pm 0.006^{\mathrm{A}}$ &, 005 \\
RPF $\left(\mathrm{g} \mathrm{g}^{-1}\right)$ & $0,671 \pm 0,005^{\mathrm{B}}$ & $0,629 \pm 0,006^{\mathrm{A}}$ & $0,661 \pm 0,006^{\mathrm{B}}$ & $<, 001$ \\
Ln RBAR $\left(\mathrm{g} \mathrm{g}^{-1}\right)$ & $1,643 \pm 0,040^{\mathrm{B}}$ & $1,459 \pm 0,041^{\mathrm{A}}$ & $1,666 \pm 0,40^{\mathrm{B}}$ &, 001 \\
AFE$^{\wedge}$ (cm g $^{-1)}$ & $0,00410 \pm 0,00011^{\mathrm{C}}$ & $0,00320 \pm$ & $0,00369 \pm$ & $<, 001$ \\
\hline
\end{tabular}

Tabla 4 Parámetros de crecimiento en función del sustrato

Diferentes letras indican diferencias significativas. LT Longitud tallo. LR Longitud raíz. PST Peso seco total, RPF Relación peso foliar, RBAR Relación biomasa aérea/ biomasa radicular, AFE Área foliar específica, $(\mathrm{n}=50$ alfa= $0.05)$ 


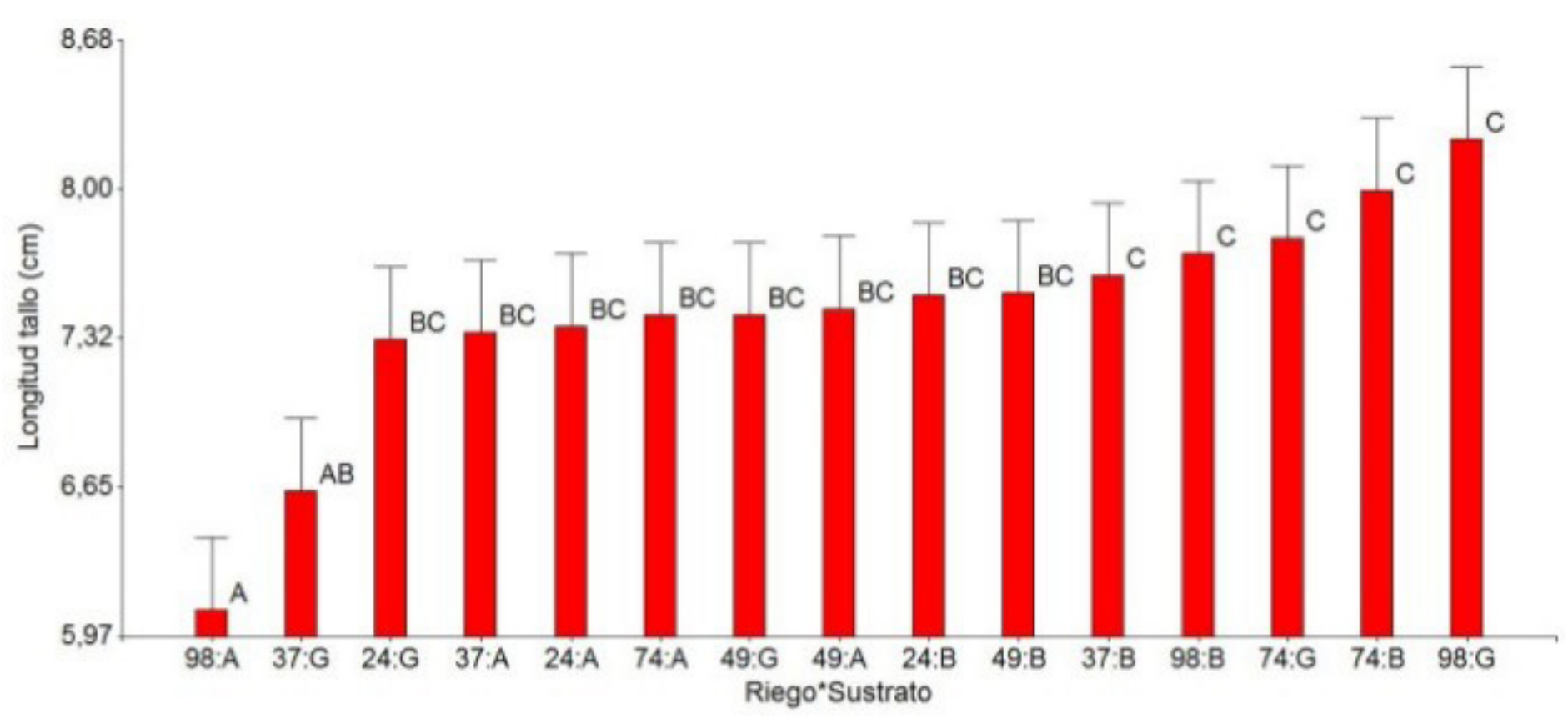

Ilustración 1: Longitud del tallo en función de la interacción de las variables riego y sustrato

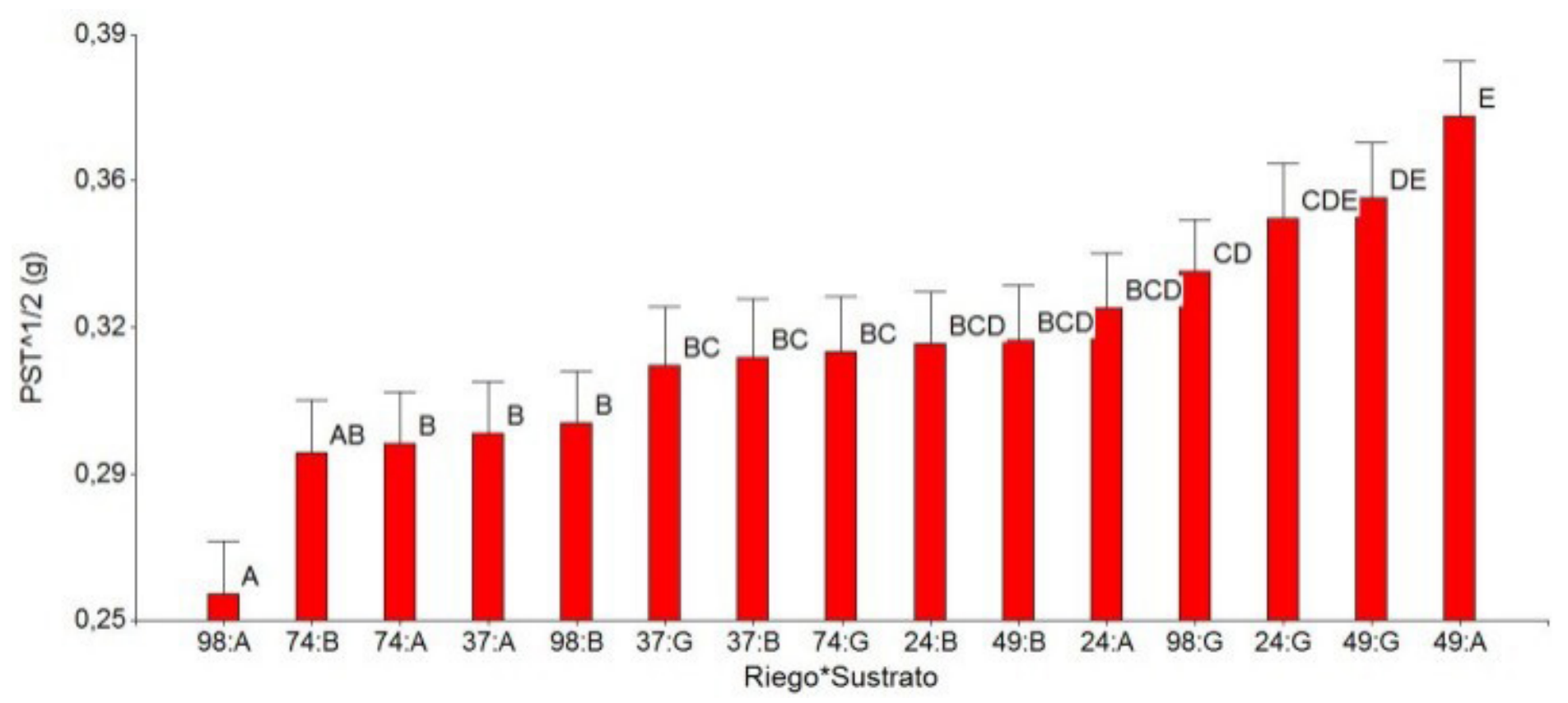

Ilustración 2: Peso seco total en función de la interacción de las variables riego y sustrato 


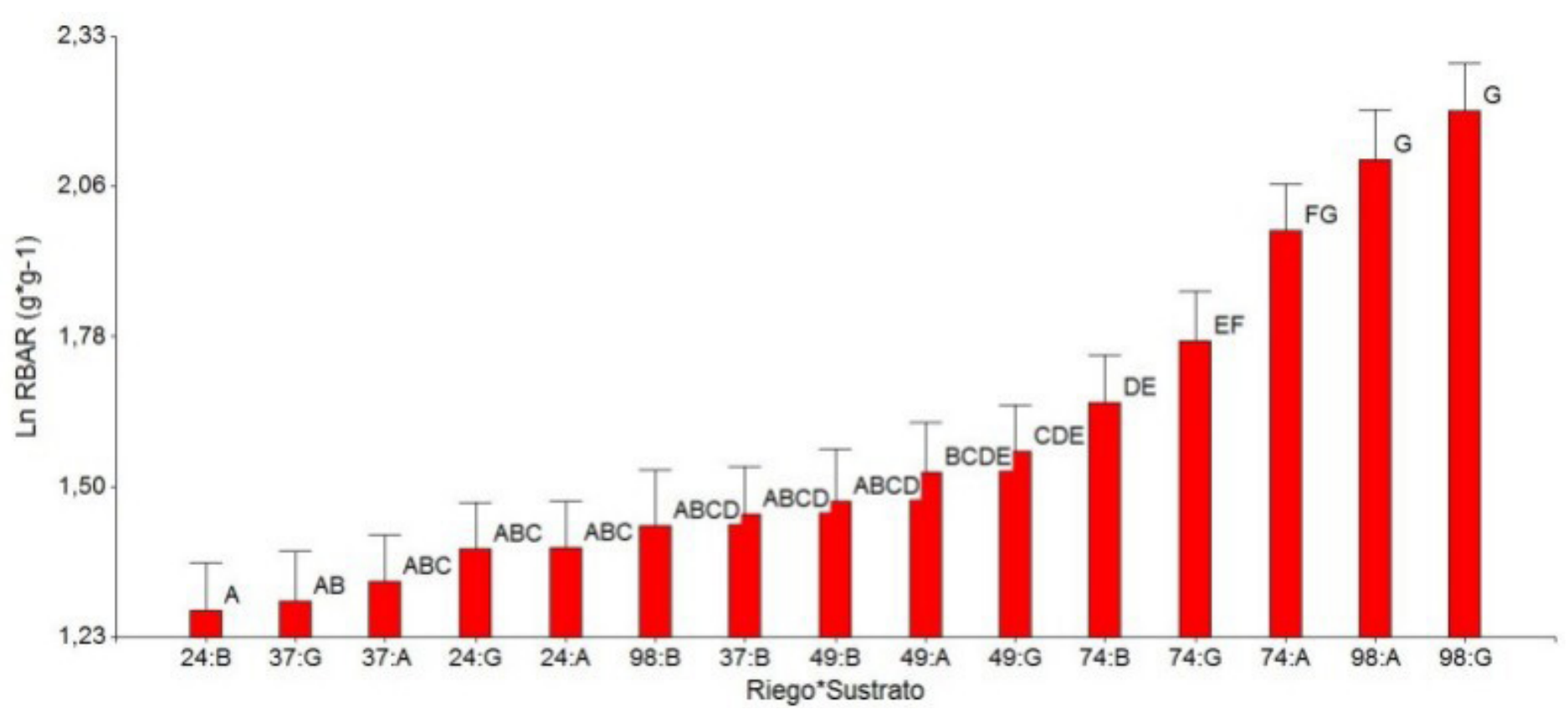

Ilustración 3: Relación peso foliar en función de la interacción de las variables riego y sustrato

\section{AGRADECIMIENTO}

Esta investigación es producto de la alianza entre la UAB y la UNAN FAREM estelí, con auspicio del proyecto "Enfoque territorial contra el cambio climático. Medidas de adaptación y reducción de vulnerabilidades en la región de Las Segovias Nicaragua" ejecutado por PNUD.

\section{BIBLIOGRAFÍA}

Bewley, J. ., \& Black, M. (n.d.). Physiology and Biochemistry of Seeds . (pp. 306-375).

Guerrero, N. R., Andrés, M., Quintero, O., \&

Pérez, C. (2012). Determinación del Área

Foliar en Fotografías Tomadas con una Cámara

Web , un Teléfono Celular o una Cámara

Semiprofesional Leaf Area Measurement in

Photographs Taken with a Webcam, a Cell Phone or a Semi Professional Camera frecuencia en la investigación, 65(1), 6399-6405.

Holdridge, \& R, L. (1979). Ecología basada en zonas de vida. San José de Costa Rica., 1979.

Janzen, D. H. (1983). Dispersal of seeds by vertebrate guts. D.J \& M Slatkin (eds.). Coevolution, 232265.

Kramer, E. A. (1997). Measuring lan dscape changes in remnant tropical dry forest. W.F. Lawrence \& R.G. Bierregaard (eds.). Tropical forest remnants: ecology, management, and conservation of fragmented communities, 386-399.

M, R., \& J.R. (2003). Viveros forestales: Cultivo de brinzales forestales en envase. Sustrato o medio de cultivo., 2003.

Miles, L., Newton, A. C., DeFries, R., Ravilious, C., May, I., Blyth, S., \& Gordon, J. E. (2006). A global overview of the conservation status of tropical dry forests. Journal of Biogeography, 33, 491- 505.

Narbona, E., Arista, M., \& Ortiz, P. L. (2003). GERMINACIÓN DE LAS SEMILLAS DEL MADROÑO (ARBUTUS UNEDO L., ERICACEAE), 73-78.

Pardos, J. A. (2004). Respuestas de las plantas al anegamiento del suelo, 101-107.

Pedroza, H. (IICA), \& Dicovskyi, L. (IICA). (2007). Sistema de análisis estadístico con SPSS (p. 152). Managua. 